\title{
Contrastive Analysis of Neuropsychology and Personality Characteristics of Male Lacunar Infarction Patients with Hazardous Drinking and Non-Hazardous Drinking
}

This article was published in the following Dove Press journal:

Neuropsychiatric Disease and Treatment

\author{
Jia Yang ${ }^{\prime}$ \\ Zhandong Qiu ${ }^{1,2}$ \\ Yu Fang ${ }^{3}$ \\ 'Department of Neurology, Xuanwu \\ Hospital of Capital Medical University, \\ Beijing I00053, People's Republic of \\ China; ${ }^{2}$ Department of Neurology, Tongji \\ Hospital Affiliated to Tongji Medical \\ College, Huazhong University of Science \\ and Technology, Wuhan 430030, People's \\ Republic of China; ${ }^{3}$ Department of \\ Emergency Medicine, Tongji Hospital \\ Affiliated to Tongji Medical College, \\ Huazhong University of Science and \\ Technology, Wuhan 430030, People's \\ Republic of China
}

\begin{abstract}
Objective: To compare the differences in neuropsychological and personality characteristics between male patients with lacunar infarction with hazardous drinking and non-hazardous drinking.
\end{abstract}

Methods: From May to October 2016, a total of 124 male outpatients and inpatients with lacunar infarction were selected in the Department of Neurology, Tongji Hospital, and they were divided into a hazardous drinking group (HD group, $n=52$ ) and a non-hazardous drinking group (NHD group, $\mathrm{n}=72$ ) according to alcohol consumption habits. General information, MoCA score, EPQ score and SCL-90 score were compared between the two groups.

Results: Incidence of cognitive disorder in the HD group and NHD group was $59.6 \%$ and $56.9 \%$ respectively, showing no significant difference $(\mathrm{P}>0.05)$. Scores of visuospatial and executive function, memory, attention and total MoCA score in the HD group were significantly lower than those in the NHD group $(\mathrm{P}<0.05)$, while no significant difference in naming, language, abstract thinking or orientation was found between the two groups $(\mathrm{P}>0.05)$. Scores of extroversion and introversion, neuroticism and psychoticism in the HD group were significantly higher than those in the NHD group $(\mathrm{P}<0.05)$, while no significant difference in lie or feint score was found between the two groups $(\mathrm{P}>0.05)$. Scores of somatization, interpersonal sensitivity, anxiety, hostility, bigotry and psychoticism factors, and total SCL-90 score in the HD group were significantly higher than those in the NHD group $(\mathrm{P}<0.05)$, while no significant difference in scores of obsessive-compulsive, depression and terror factors were found between the two groups $(\mathrm{P}>0.05)$.

Conclusion: Compared with male patients with lacunar infarction with non-hazardous drinking, male lacunar infarction patients with hazardous drinking showed worse visuospatial and executive function, memory, attentiveness, cognitive function and mental health status, with more obvious change of personality, thus extra attention is needed for male lacunar infarction patients with hazardous drinking.

Keywords: male, lacunar infarction, hazardous drinking, neuropsychology, personality

Studies have shown that chronic alcohol abuse affects the structure and function of the nervous system, resulting in neuropsychiatric symptoms such as cognitive impairment, emotional disorders, sensory and motor dysfunction, and negative impact on the personality and psychological state of patients. ${ }^{1,2}$ Hazardous drinking is one of the most major risks for cardiovascular and cerebrovascular diseases, with average weekly or daily alcohol intake exceeding safe limits. Lacunar infarction
Correspondence: Zhandong Qiu Email003qiu86@I63.com 
accounts for nearly one-third of ischemic strokes, ${ }^{3}$ but there are few reports on the effects of hazardous drinking on neuropsychology and personality characteristics of lacunar infarction patients. This study aims to compare and analyze the differences in neuropsychological and personality characteristics of male lacunar infarction patients with hazardous drinking and non-hazardous drinking, so as to improve the awareness of male lacunar infarction patients on the importance of abstinence.

\section{Objects and Methods Objects}

A total of 124 male outpatients and inpatients with lacunar infarction from the Department of Neurology, Tongji Hospital Affiliated to Tongji Medical College, Huazhong University of Science and Technology were selected and divided into hazardous drinking group (HD group) with 52 cases and nonhazardous drinking group (NHD group) with 72 cases according to their alcohol consumption habits. Inclusion criteria were: (1) Age $\geq 45$ years old; (2) Meet the diagnostic criteria of lacunar infarction ${ }^{4}$ with diagnosis within 1 week of onset; (3) Craniocerebral magnetic resonance imaging (MRI) results meet the imaging diagnostic criteria for lacunar infarction excepting those with extensive white matter demyelination (bilateral flaky or multifocal lesions, mostly located around the ventricle, centrum semiovale, deep white matter and optic radiation, which is described by the radiologist based on the imaging findings); (4) Those with clear consciousness, no obvious speech disorders; (5) Those who can take care of themselves; (6) Patients with hypertension; (7) Patients and their families voluntarily participate in the study after informed interviews, and cooperate in the interviews and complete the scales. Exclusion criteria were: (1) Patients with primary respiratory, digestive, cardiovascular, blood, immune system chronic diseases or definite malignant tumors; (2) Non-alcohol-related intellectual or mental disorders; (3) Patients with clear history of cerebral hemorrhage, cerebral infarction, brain tumor, thyroid dysfunction or brain surgery; (4) Those who have taken or are taking folic acid or vitamin B12 and drugs that may affect cognitive function, mood and mental symptoms or drug abusers; (5) Those with severe consciousness disturbance, visual impairment, hearing impairment, or speech disorder.

\section{Ethics}

This study involving people and medical records, in full accordance with the Declaration of Helsinki, was approved by the medical ethical review committee of Tongji Hospital, and all patients provided written informed consent prior to participation. Participants were informed that participation in the trial was absolutely voluntary, and that they could withdraw from the trial at any time. Research investigators fully complied with Good Clinical Practice guidelines. ${ }^{5}$

\section{Hazardous Drinking}

Referring to the Alcohol Abuse and Alcoholism Screening Guide, ${ }^{6}$ hazardous drinking was defined as follows: more than 14 standard amounts per week or more than 4 standard amounts at one time, of which one standard amount is $12 \mathrm{~g}$ pure alcohol, namely $180 \mathrm{~mL}$ wine or $360 \mathrm{~mL}$ beer or $45 \mathrm{~mL}$ of distilled spirits.

\section{Observed Indicators}

General information of the two groups (including age, education level, occupation type, smoking history, parents' drinking, digestive system diseases, cardiovascular system diseases, peripheral neuropathy and BMI) was collected, MoCA, EPQ and SCL-90 were scored by interviews, and all data were recorded by computer.

\section{MoCA (Montreal Cognitive Assessment) ${ }^{7}$}

The MoCA covers 7 aspects, i.e. visuospatial and executive function, naming, memory, attention, language, abstract thinking and orientation, with a full score of 30 . MoCA $\geq 26$ indicates normal cognitive function and $<26$ indicates cognitive impairment (MoCA plus 1 point for subjects with less than 12 years of education). The lower the score, the worse the cognitive function. The research results of similar populations in China show that the MoCA has a reliability of 0.95 and a validity of $0.85 .^{8}$

\section{EPQ (Eysenck Personality Questionnaire) ${ }^{9}$}

The EPQ is currently one of the most widely used personality analysis questionnaires in the fields of medicine, education, psychology and justice, practical for all kinds of people. It is based on three basic factors of personality, namely extroversion and introversion $(\mathrm{E})$, neuroticism $(\mathrm{N})$ and psychoticism (P). E is mainly to evaluate internal and external tendency: high $\mathrm{E}$ indicates extroverted, outgoing, sociable and adventurous, low E indicates introverted, orderly, quiet; $\mathrm{N}$ mainly shows emotional stability tendency: high $\mathrm{N}$ indicates anxiety, worry, emotional and irrational, low $\mathrm{N}$ indicates stable, selfcontrol, slow and mild emotional response; while low $\mathrm{P}$ is considered to be sympathetic, and concerned about others, and high P mainly reflects characteristics of indifference, 
celibacy, cruelty, hostility. In addition, lie (L) is a measure for subjects of concealing their true thoughts or social naive level, high $\mathrm{L}$ indicates hiding their true thoughts.

\section{SCL-90 (SymptomChecklist90) ${ }^{9}$}

The SCL-90 is the most widely used psychological scale in the examination of mental disorders and mental diseases. It consists of 90 items, including 9 elements for different symptoms, namely somatization, obsessive-compulsive, interpersonal sensitivity, depression, anxiety, hostility, terror, bigotry and psychoticism. The psychological state of the subjects can be evaluated from the dimensions of thinking, emotion, behavior, interpersonal relationship and living habits. The greater the number of positive items, the higher the score for each factor, with a higher score indicating worse psychological health. The score for each factor reflects the severity of symptoms, and the total average score gives the general symptomatic index, which can show the mental status of the subjects as a whole. The reliability and validity meet the requirements of measurement.

\section{Statistical Analyses}

The measurement data which accord with normal distribution were expressed as $\mathrm{x} \pm \mathrm{s}$ and $t$-test was used for two independent samples. Measurement data that do not conform to normal distribution were expressed by $\mathrm{M}(\mathrm{QR})$, and rank sum test was adopted. Count data were expressed as percentages and $\chi^{2}$ test or Fisher's exact test was used. Statistical analysis was performed using SPSS 21.0 (IBM SPSS, Armonk, NY, USA). $\mathrm{P}<0.05$ was considered to indicate a statistically significant difference.

\section{Results}

\section{General Information}

There was no significant difference in age, education level, occupation type, digestive system diseases, cardiovascular system diseases and BMI between the two groups. The two groups had statistically significant differences in terms of smoking history, parents' drinking and peripheral neuropathy (Table 1).

\section{MoCA}

The incidence of cognitive impairment was $59.6 \%$ in the HD group and $56.9 \%$ in the NHD group, with no statistically significant difference $\left(\chi^{2}=0.088, P=0.766\right)$. The scores of visuospatial and executive function, memory, attention and total MoCA of patients in the HD group were lower than those in the NHD group, and the difference was significant. There was no significant difference in the scores of naming, language, abstract thinking and orientation between the two groups (Table 2).

\section{EPQ}

The scores of $\mathrm{E}, \mathrm{N}$ and $\mathrm{P}$ in the HD group were higher than those in the NHD group, with statistically significant differences. There was no statistically significant difference in the score of $\mathrm{L}$ between the two groups (Table 3).

\section{SCL-90}

The scores of somatization, interpersonal sensitivity, anxiety, hostility, bigotry, psychoticism and the total average of SCL-90 in the HD group were higher than those in the NHD group, and the difference was statistically significant. There was no statistically significant difference in scores of obsessive-compulsive, depression and terror between the two groups (Table 4).

\section{Discussion}

Studies have shown that chronic alcoholism can be caused by long-term excessive drinking, and the direct and indirect toxicity of alcohol can damage the central and peripheral nerves and muscle tissue, causing chronic mental health symptoms, extensive and severe nervous system damage, and eventually causing neuropsychiatric symptoms such as epilepsy, dementia, tremor, delirium, illusion, delusion, ataxia, peripheral sensory disturbance, weakness, etc. ${ }^{10-12}$ Moreover, chronic alcohol abuse can also change the personality, increase the incidence of anxiety and emotional disorders, affecting the mental health of drinkers, and some drinkers may even commit suicide or engage in criminal behavior. ${ }^{13}$ For patients with lacunar infarction, hazardous drinking not only plays the role of "cause" (risk factor), but also has a complex impact on their neuropsychology and personality characteristics due to the longterm damage to the nervous system, psychological basis and characteristics of alcoholic behavior.

Several studies have shown that moderate drinking can reduce the risk of dementia, ${ }^{12,14-16}$ but chronic alcohol abuse will cause cognitive decline, and cognitive impairment may be restored after abstinence, indicating that nervous tissue is plastic and alcohol-induced injuries of the nervous system are often reversible. ${ }^{17-19}$ In this study, MoCA was used to evaluate cognitive function. The results showed that compared with the NHD group, patients in the HD group had lower scores in visuospatial and executive function, memory, attention and the total scores of MoCA, 
Table I Comparison of General Information Between the Two Groups

\begin{tabular}{|c|c|c|c|c|}
\hline & $H D(n=52)$ & NHD $(n=72)$ & $\chi^{2}$ & $P$ \\
\hline Age, years & & & 0.106 & 0.948 \\
\hline $45 \sim 59$ & $22(42.3)$ & $31(43.1)$ & & \\
\hline $60 \sim 74$ & $24(46.2)$ & $34(47.2)$ & & \\
\hline$\geq 75$ & $6(11.5)$ & $7(9.7)$ & & \\
\hline Education level & & & 1.675 & 0.433 \\
\hline$\leq$ Primary school & II (2I.I) & $19(26.4)$ & & \\
\hline Middle school & $34(65.4)$ & $48(66.7)$ & & \\
\hline$\geq$ University degree & $7(\mid 3.5)$ & $5(6.9)$ & & \\
\hline Occupational type & & & 1.179 & 0.278 \\
\hline Manual labor & $32(61.5)$ & $51(70.8)$ & & \\
\hline Mental work & $20(38.5)$ & $21(29.2)$ & & \\
\hline Smoking history & & & 4.416 & 0.036 \\
\hline Yes & $18(34.6)$ & $13(18.1)$ & & \\
\hline No & $34(65.4)$ & $59(81.9)$ & & \\
\hline Parents' drinking & & & 17.007 & $<0.001$ \\
\hline Unilateral drinking & $35(67.3)$ & $27(37.5)$ & & \\
\hline Bilateral drinking & $5(9.6)$ & $2(2.8)$ & & \\
\hline No drinking & $12(23.1)$ & $43(59.7)$ & & \\
\hline Digestive system diseases & $21(40.4)$ & $18(25.0)$ & 3.315 & 0.069 \\
\hline Cardiovascular system diseases & $23(44.2)$ & $28(38.9)$ & 0.356 & 0.551 \\
\hline Peripheral neuropathy & $24(46.2)$ & $16(22.2)$ & 7.913 & 0.005 \\
\hline BMI & & & 6.168 & 0.095 \\
\hline$<18.5$ & $3(5.8)$ & $3(4.2)$ & & \\
\hline$|8.5 \leq \mathrm{BM}|<24$ & $35(67.3)$ & $37(51.4)$ & & \\
\hline $24 \leq \mathrm{BM} \mid<28$ & $13(25.0)$ & $23(31.9)$ & & \\
\hline$\geq 28$ & I (I.9) & $9(12.5)$ & & \\
\hline
\end{tabular}

Note: Data are presented as $\mathrm{n}(\%)$.

indicating that the visuospatial and executive function, memory and attention of male patients with lacunar infarction were damaged seriously, and their cognitive function was poor. But there is no statistical difference between the

Table 2 Comparison of MoCA Score Between the Two Groups ( $M(\mathrm{QR})$, Score)

\begin{tabular}{|l|l|l|l|l|}
\hline & $\begin{array}{l}\text { HD } \\
(\mathbf{n = 5 2 )}\end{array}$ & $\begin{array}{l}\text { NHD } \\
(\mathbf{n = 7 2 )}\end{array}$ & $\mathbf{Z}$ & $\boldsymbol{P}$ \\
\hline $\begin{array}{l}\text { Visuospatial and } \\
\text { executive }\end{array}$ & $4(2)$ & $4(I)$ & -2.342 & 0.019 \\
Naming & $3(I)$ & $3(I)$ & -1.26 & 0.208 \\
Memory & $4(2)$ & $4(2)$ & -2.184 & 0.029 \\
Attention & $5(2)$ & $6(I)$ & -2.2 & 0.028 \\
Language & $2(I)$ & $2(I)$ & -0.594 & 0.553 \\
Abstract thinking & $2(I)$ & $2(I)$ & -1.3 & 0.194 \\
Orientation & $5(2)$ & $6(I)$ & -1.733 & 0.083 \\
Total & $25(4)$ & $25(4)$ & -2.686 & 0.007 \\
\hline
\end{tabular}

Note: Data are presented as $M(Q R)$, score. two groups in the cognitive impairment incidence and the scores of naming, language, abstract thinking, and orientation. These results may be related to the small sample size; a large sample, multicenter, age stratified study is warranted.

Previous studies have shown that chronic alcohol abuse can change the personality, manifesting for example as showing decreased responsibility and morality, less concern for family and work, burnout and inaction, and being more self-centered. ${ }^{8,20}$ EPQ was used for personality

Table 3 Comparison of EPQ Score Between the Two Groups

\begin{tabular}{|l|l|l|l|l|}
\hline & HD (n=52) & NHD (n=72) & $Z$ & $P$ \\
\hline E & $63(15)$ & $60(15)$ & -2.239 & 0.025 \\
N & $60(10)$ & $55(15)$ & -2.058 & 0.040 \\
P & $65(20)$ & $65(20)$ & -1.978 & 0.048 \\
L & $35(15)$ & $35(10)$ & -0.753 & 0.452 \\
\hline
\end{tabular}

Note: Data are presented as $M(Q R)$, score. 
Table 4 Comparison of SCL-90 Score Between the Two Groups

\begin{tabular}{|c|c|c|c|c|}
\hline & $H D(n=52)$ & NHD $(n=72)$ & $Z(t)$ & $P$ \\
\hline Somatization [M (QR)] & $1.92(0.48)$ & $1.75(0.64)$ & -3.122 & 0.002 \\
\hline Obsessive-compulsive [M (QR)] & $2.20(1.25)$ & $2.05(0.80)$ & -1.616 & 0.106 \\
\hline Interpersonal sensitivity $(x \pm s)$ & $2.52 \pm 0.58$ & $2.22 \pm 0.45$ & $3.258^{\mathrm{a}}$ & 0.001 \\
\hline Depression [M (QR)] & $1.65(0.46)$ & $1.69(0.68)$ & -0.378 & 0.706 \\
\hline Anxiety ( $x \pm s)$ & $2.16 \pm 0.50$ & $1.96 \pm 0.49$ & $2.199^{\mathrm{a}}$ & 0.030 \\
\hline Hostility $(x \pm s)$ & $2.46 \pm 0.5 \mathrm{I}$ & $2.27 \pm 0.42$ & $2.188^{\mathrm{a}}$ & 0.031 \\
\hline Terror [M (QR)] & $2.00(0.57)$ & $1.86(0.54)$ & -0.916 & 0.360 \\
\hline Bigotry [M (QR)] & $2.00(0.83)$ & $1.83(0.46)$ & -3.289 & 0.001 \\
\hline Psychoticism [M (QR)] & $2.10(0.90)$ & $1.70(0.70)$ & -3.917 & $<0.001$ \\
\hline Total average score $(x \pm s)$ & $2.10 \pm 0.18$ & $1.93 \pm 0.17$ & $5.54 I^{\mathrm{a}}$ & $<0.001$ \\
\hline
\end{tabular}

Note: ${ }^{a}$ Indicates t value.

analysis in this study. The results showed that the E, N and $\mathrm{P}$ scores of patients in the HD group were higher than those in the NHD group, suggesting that male patients with lacunar infarction with hazardous drinking have obvious personality changes compared with those patients with non-hazardous drinking. Patients in the HD group were often sociable but prone to loneliness, hostility to people, pursuit of stimulation and adventure, impulsiveness, carelessness, rapid and strong response, without control of emotions, suffering from various psychosomatic disorders, and having difficulty to truly adapt to the environment and to others while desiring attention.

Somatization in SCL-90 refers to physical manifestations such as headache, back pain, muscle soreness and other physical discomfort of cardiovascular, digestive and respiratory systems. The sensitivity of interpersonal relationship mainly refers to discomfort and inferiority in interpersonal communication. Anxiety refers to dysphoria, nervousness and the resulting physical signs such as tremor, etc. Hostility includes hostile thoughts, feelings and behaviors. Bigotry mainly refers to projection thinking, hostility, suspicion, delusion, etc. Psychoticism levels are the main indicators to reflect psychotic behavior and lifestyle. In this study, SCL-90 was used to evaluate the status of mental health. The results showed that the scores of somatization, interpersonal sensitivity, anxiety, hostility, bigotry, psychoticism and total average scores of SCL-90 in the HD group were higher than those in the NHD group, suggesting that the above indicators of male patients with lacunar infarction with hazardous drinking were more severe and their mental health status was poorer than that of those patients with non-hazardous drinking.

To sum up, compared with male patients with lacunar infarction with non-hazardous drinking, patients with hazardous drinking have serious impairment of visuospatial and executive function, memory and attention, obvious personality changes, poor cognitive function and mental health status. Therefore, in clinical application, we should pay extra attention to male patients with lacunar infarction with hazardous drinking, and initiate necessary medical and social psychological intervention, so as to improve their treatment compliance, therapeutic efficacy and long-term prognosis.

\section{Disclosure}

The authors report no conflicts of interest in this work.

\section{References}

1. Shield KD, Parry C, Rehm J. Chronic diseases and conditions related to alcohol use. Alcohol Res. 2013;35(2):155-173.

2. Levola J, Aalto M, Holopainen A, Cieza A, Pitkanen T. Health-related quality of life in alcohol dependence: a systematic literature review with a specific focus on the role of depression and other psychopathology. Nord $J \quad$ Psychiatry. 2014;68(6):369-384. doi:10.3109/08039488.2013.852242

3. Regenhardt RW, Das AS, Lo EH, Caplan LR. Advances in understanding the pathophysiology of lacunar stroke: a review. JAMA Neurol. 2018;75(10):1273-1281. doi:10.1001/jamaneurol.2018.1073

4. Charidimou A, Pantoni L, Love S. The concept of sporadic cerebral small vessel disease: a road map on key definitions and current concepts. Int J Stroke. 2016;11(1):6-18. doi:10.1177/ 1747493015607485

5. ICH harmonized tripartite guideline. Guideline for Good Clinical Practice. J Postgrad Med. 2001;47(1):45-50.

6. Parast L, Meredith LS, Stein BD, Shadel WG, D'Amico EJ. Identifying adolescents with alcohol use disorder: optimal screening using the National Institute on Alcohol Abuse and Alcoholism screening guide. Psychol Addict Behav. 2018;32(5):508-516. doi:10.1037/ adb0000377

7. Nasreddine ZS, Phillips NA, Bédirian V, et al. The Montreal Cognitive Assessment, MoCA: a brief screening tool for mild cognitive impairment. J Am Geriatr Soc. 2005;53(4):695-699. doi:10.1111/ j.1532-5415.2005.53221.x

8. Chu LW, Ng KH, Law AC, Lee AM, Kwan F. Validity of the Cantonese Chinese Montreal Cognitive Assessment in Southern Chinese. Geriatr Gerontol Int. 2015;15(1):96-103. doi:10.1111/ ggi. 12237 
9. Wei Y, Li H, Wang H, Zhang S, Sun Y. Psychological Status of Volunteers in a Phase I Clinical Trial Assessed by Symptom Checklist 90 (SCL-90) and Eysenck Personality Questionnaire (EPQ). Med Sci Monit. 2018;24:4968-4973. doi:10.12659/ MSM.909524

10. Streissguth AP, Landesman-Dwyer S, Martin JC, Smith DW. Teratogenic effects of alcohol in humans and laboratory animals. Science. 1980;209(4454):353-361. doi:10.1126/science.6992275

11. Randall-James J, Wadd S, Edwards K, Thake A. Alcohol screening in people with cognitive impairment: an exploratory study. J Dual Diagn. 2015;11(1):65-74. doi:10.1080/15504263.2014.992095

12. Davis BJ, Vidal JS, Garcia M, et al. The alcohol paradox: light-tomoderate alcohol consumption, cognitive function, and brain volume. J Gerontol a Biol Sci Med Sci. 2014;69(12):1528-1535. doi:10.1093/ gerona/glu092

13. Kaila-Kangas L, Koskinen A, Leino-Arjas P, Virtanen M, Harkanen T, Lallukka T. Alcohol use and sickness absence due to all causes and mental- or musculoskeletal disorders: a nationally representative study. BMC Public Health. 2018;18(1):152. doi:10.1186/s12889-018-5059-8

14. Topiwala A, Allan CL, Valkanova V, et al. Moderate alcohol consumption as risk factor for adverse brain outcomes and cognitive decline: longitudinal cohort study. BMJ. 2017;357:j2353. doi: $10.1136 /$ bmj.j2353
15. Rehm J, Hasan OSM, Black SE, Shield KD, Schwarzinger M. Alcohol use and dementia: a systematic scoping review. Alzheimers Res Ther. 2019;11(1):1. doi:10.1186/s13195-018-0453-0

16. Mulhauser K, Weinstock J, Ruppert P, Benware J. Changes in neuropsychological status during the initial phase of abstinence in alcohol use disorder: neurocognitive impairment and implications for clinical care. Subst Use Misuse. 2018;53(6):881-890. doi:10.1080/ 10826084.2017.1408328

17. Seo D, Sinha R. Neuroplasticity and predictors of alcohol recovery. Alcohol Res. 2015;37(1):143-152.

18. Creswell KG, Chung T. Treatment for alcohol use disorder: progress in predicting treatment outcome and validating nonabstinent end points. Alcohol Clin Exp Res. 2018;42(10):1874-1879. doi:10.1111/ acer. 13846

19. Ros-Cucurull E, Palma-Alvarez RF, Cardona-Rubira C, et al. Alcohol use disorder and cognitive impairment in old age patients: a 6 months follow-up study in an outpatient unit in Barcelona. Psychiatry Res. 2018;261:361-366. doi:10.1016/j.psychres.2017.12.069

20. McCarter KL, Halpin SA, Baker AL, et al. Associations between personality disorder characteristics and treatment outcomes in people with co-occurring alcohol misuse and depression. BMC Psychiatry. 2016;16:210. doi:10.1186/s12888-016-0937-z
Neuropsychiatric Disease and Treatment

\section{Publish your work in this journal}

Neuropsychiatric Disease and Treatment is an international, peerreviewed journal of clinical therapeutics and pharmacology focusing on concise rapid reporting of clinical or pre-clinical studies on a range of neuropsychiatric and neurological disorders. This journal is indexed on PubMed Central, the 'PsycINFO' database and CAS, and is the official journal of The International Neuropsychiatric Association (INA). The manuscript management system is completely online and includes a very quick and fair peer-review system, which is all easy to use. Visit http://www.dovepress.com/testimonials.php to read real quotes from published authors. 